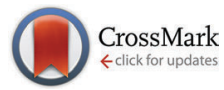

Cite this: Phys. Chem. Chem. Phys., 2016, 18, 24015

Received 6th May 2016 Accepted 18th July 2016 DOI: 10.1039/c6cp03072f

www.rsc.org/pccp

\title{
Separation of dynamic and nondynamic correlation
}

\author{
Eloy Ramos-Cordoba, ${ }^{\star a b}$ Pedro Salvador ${ }^{c}$ and Eduard Matito*ad
}

\begin{abstract}
The account of electron correlation and its efficient separation into dynamic and nondynamic parts plays a key role in the development of computational methods. In this paper we suggest a physicallysound matrix formulation to split electron correlation into dynamic and nondynamic parts using the two-particle cumulant matrix and a measure of the deviation from idempotency of the first-order density matrix. These matrices are applied to a two-electron model, giving rise to a simplified electron correlation index that (i) depends only on natural orbitals and their occupancies, (ii) can be straightforwardly decomposed into orbital contributions and (iii) splits into dynamic and nondynamic correlation parts that (iv) admit a local version. These expressions are shown to account for dynamic and nondynamic correlation in a variety of systems containing different electron correlation regimes, thus providing the first separation of dynamic and nondynamic correlation using solely natural orbital occupancies.
\end{abstract}

\section{Introduction}

The account of electron correlation effects-i.e., the interaction between electrons in a quantum system -is still a most important challenge in current computational chemistry. ${ }^{1-4}$ Many different properties are affected by electron correlation, including bond stretching and dissociation, electron delocalization or antiferromagnetic interactions. The issue has been addressed from multiple perspectives, giving rise to a manifold of methods to calculate the energy of a molecular system. Each method has its own way to tackle the problem and, to some extent, recover electron correlation effects. There are multiple phenomena behind electron correlation, which have been largely studied in the past sixty years. ${ }^{2-10}$ As a result, nowadays terms such as short-range, longrange, dynamic, ${ }^{11}$ nondynamic, static, ${ }^{12}$ left-right, in-out, radial or angular $^{13}$ correlation belong to the jargon that computational chemists use to analyze the missing electron correlation effects of a given method in the description of quantum systems.

Perhaps the most well-known classification of electron correlation types is done in terms of dynamic and nondynamic electron correlation. ${ }^{11} \mathrm{~A}$ method is said to include dynamic correlation if its wavefunction, calculated as a configuration interaction (CI) expansion, includes a most dominant configuration

\footnotetext{
${ }^{a}$ Faculty of Chemistry, University of the Basque Country UPV/EHU, and Donostia International Physics Center (DIPC)., P.K. 1072, 20080 Donostia, Euskadi, Spain. E-mail: eloy.raco@gmail.com, ematito@gmail.com

${ }^{b}$ Department of Chemistry, University of California Berkeley, Berkeley, California 94720, USA

c Institut de Quimica Computacional i Catàlisi (IQCC) and Departament de Quimica, University of Girona, 17071 Girona, Catalonia, Spain

${ }^{d}$ IKERBASQUE, Basque Foundation for Science, 48011 Bilbao, Spain
}

and only small (but energetically important) contributions of other configurations. Conversely, nondynamic correlation arises when there are two or more important configurations, usually in the presence of degeneracies or near-degeneracies. Nondynamic correlation is regarded as a system-specific contribution, whereas dynamic electron correlation is accepted to be a rather universal contribution. ${ }^{11,14}$ Some authors ${ }^{12}$ distinguish between nondynamic and static correlation and define static correlation as the nondynamic correlation required to provide a correct zeroth-order description as dictated by spin and symmetry considerations, whereas nondynamic correlation is reserved for other situations such as the separation of molecules into fragments or the description of some excited states. In this paper we will use the term nondynamic correlation in a wide sense, comprising both static and (pure) nondynamic effects, i.e., all the electron correlation that is not dynamic. Interestingly, many popular methods introduce mainly either one or the other correlation type, and therefore, a large number of computational approaches can be classified as either dynamic- or nondynamic-correlation including methods. For instance, truncated CI and coupled-cluster (CC) wavefunctions give a good account of dynamic correlation, while multiconfiguration (MC) wavefunctions introduce mostly nondynamic correlation. Back in the 90's different diagnostic tools were put forward in order to evaluate the extent of dynamic and nondynamic correlation included in computational methods. For instance, the so-called T1 diagnostic of Lee and coworkers, ${ }^{15,16}$ the less-known D1 diagnostic ${ }^{17}$ and subsequent modifications ${ }^{18}$ have been widely used to measure the importance of nondynamic correlation in coupled-cluster wave functions. Cioslowski also developed the differential density matrix overlap (DDMO) for the assessment of electron correlation effects. ${ }^{19}$ The development 
of new cost-efficient computational methods has often consisted in the inclusion of dynamic or nondynamic correlation to an existing approach that already incorporates the other correlation type. This has given rise to a plethora of procedures such as second-order perturbation theory from complete active space wavefunctions (CASPT2), ${ }^{20}$ multireference configuration interactions with single and double excitations (MRCI-SD) ${ }^{21}$ or the antihermitian contracted Schrödinger equation (ACSE). ${ }^{22,23}$ The mixing of two existing computational approximations has also resulted in the construction of hybrid methods, often combining density functional theory (DFT) and wavefunction techniques. $^{24,25}$ Range-separated methods ${ }^{26}$ which provide a scheme to merge short and long-range correlation, or (local) ${ }^{27}$ hybrid density functionals, ${ }^{28,29}$ which use orbital-based HartreeFock exchange mixed with DFT exchange are just two examples. These methods use optimally-chosen mixing parameters or local mixing functions (LMF) to ponderate the ingredients combined. The mixture of exact exchange and density functional approximations should be determined according to the properties of each system $^{30}$ or, even better, using a LMF that depends on the features of the target molecule. ${ }^{27,31}$ The admixture is rationalized as a balance between the simulation of long-range nondynamic correlation effects (included by the DFT exchange, usually through the generalized-gradient approximation) and the selfinteraction correction (exact exchange), ${ }^{2}$ whereas the inclusion of dynamic correlation effects is given by a local correlation functional multiplied by a mixing parameter. Range-separated functionals ${ }^{32}$ depend on attenuating parameters that have been likewise shown to be system specific. ${ }^{33}$ The key question in local hybrid functionals is the choice of the LMF, determining the local relative contributions of exact and DFT exchange. Several possibilities have been explored, ${ }^{34,35}$ including the ratio of von Weizsäcker and the exact kinetic energy densities ${ }^{27}$ and the correlation length, ${ }^{36}$ which are related to the local contribution to electron correlation. ${ }^{37}$ Despite the promising results obtained, no optimal LMF providing good results in both thermodynamics and kinetic benchmark tests has been found thus far. ${ }^{36,38}$

The account of electron correlation and its efficient separation into dynamic and nondynamic effects thus plays a key role in many situations. Grimme et al. have recently put forward a local measure of nondynamic correlation based on fractional orbital occupations within finite-temperature DFT, ${ }^{39}$ Reiher and coworkers suggested orbital entaglement measures to evaluate the nondynamic correlation in density-matrix renormalization group

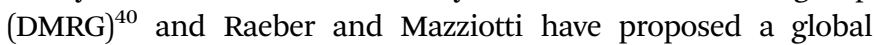
indicator of the off-diagonal long-range order correlation. ${ }^{41}$ To our knowledge, it does not exist a measure of electron correlation that can be split into dynamic and nondynamic counterparts and admits a local decomposition. Such indicator can be obviously used to assess the relative importance of dynamic and nondynamic correlation effects but it can also aid the development of new computational methods either by exploring the local character of dynamic electron correlation in local methods ${ }^{42,43}$ or by affording a new means to mix the components of hybrid methods. ${ }^{26}$ In this paper we develop a general expression to account for electron correlation effects that can be decomposed into dynamic and nondynamic parts. The expression is applied to a two-electron model, giving rise to a simplified expression that (i) depends only on natural orbitals and their occupancies, (ii) can be straightforwardly decomposed into orbital contributions, (iii) can be split into dynamic and nondynamic correlation contributions and (iv) all its contributions admit a local version. This expression is finally shown to account for dynamic and nondynamic correlation in several molecular systems, thus validating its applicability beyond the model system.

\section{Precedents}

It is not straightforward, and perhaps even impossible, to make a clear-cut separation between dynamic and nondynamic electron correlation. ${ }^{44}$ The increase of electron excitations in a CI or CC wavefunction eventually introduces some nondynamic correlation effects, whereas the increase of the number of configurations in a MC wavefunction at some point should also include dynamic correlation. However, since the source of these electron correlation types (and, therefore the way to account for them) is so different, it becomes essential to have simple expressions to distinguish one from the other. Unfortunately, there are few simple computational expressions in the literature that can provide a quantitative analysis of dynamic and nondynamic correlation effects. One of the first such separations is due to Cioslowski ${ }^{45}$ and it was, subsequently, generalized by Ludeña and coworkers. ${ }^{46,47}$ Fig. 1 summarizes the main idea. The uppermost left corner corresponds to the Hartree-Fock (HF) energy, which is connected to the opposite corner-the nonrelativistic full-CI (FCI) energy-by the correlation energy. One can also cover the distance between HF and FCI in two steps by following two different paths,

$$
E_{\mathrm{CORR}}=E_{\mathrm{ND}}^{(\mathrm{I})}+E_{\mathrm{D}}^{(\mathrm{I})}=E_{\mathrm{D}}^{(\mathrm{II})}+E_{\mathrm{ND}}^{(\mathrm{II})} .
$$

Cioslowski $^{45}$ suggested to calculate the FCI energy with a density-constrained approach that selects the FCI wavefunction reproducing the $\mathrm{HF}$ density, i.e., $E^{\mathrm{FCI}}\left[\rho^{\mathrm{HF}}\right]$. The step from $E^{\mathrm{HF}}$ to $E^{\mathrm{FCI}}\left[\rho^{\mathrm{HF}}\right]$ corresponds to path $d_{\mathrm{I}}$ in Fig. 1 , and it is expected to retrieve only the dynamic correlation because it uses the expression for an exact wavefunction but its electron density is restricted to the $\mathrm{HF}$ one. On the contrary, the relaxation process that permits global changes in the wavefunction as the electron density transforms from HF density to FCI one (path $n d_{\mathrm{I}}$ ), should account for nondynamic correlation. Ludeña suggested

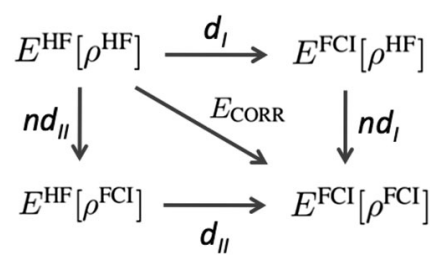

Fig. 1 Correlation energy diagram. Path I follows Cioslowski's decomposition of the electron correlation and path $I I$ is due to Ludeña and coworkers. Adapted from ref. 46. 
the path II that goes through $E^{\mathrm{HF}}\left[\rho^{\mathrm{FCI}}\right]$, which is a HF calculation using a single-determinant wavefunction restricted to reproduce the FCI density. Path II recovers first nondynamic and afterwards dynamic correlation. Cioslowski's definition is preferred because it provides a decomposition of electron correlation into nonpositive contributions, whereas Ludeña's definition provides nonnegative electron correlation values, namely, $n d_{\text {II }}>0$. Notwithstanding, both approaches afford an exact decomposition of the electron correlation energy and provide a similar description of dynamic and nondynamic correlation. ${ }^{46,47}$ Despite the physically-soundness of these methods, in practice, they require expensive FCI calculations that prevent their application beyond few-electron species. A more practical realization of dynamic/nondynamic separation is due to Handy and coworkers, ${ }^{48}$ who suggested that a fullvalence complete active space self-consistent field (CASSCF) calculation could be used as a reference wavefunction that only includes the nondynamic correlation energy, giving rise to the following separation of dynamic and nondynamic correlation energy,

$$
\begin{aligned}
E_{\mathrm{CORR}}=E_{\mathrm{FCI}}- & E_{\mathrm{HF}}=E_{\mathrm{ND}}^{\mathrm{H}}+E_{\mathrm{D}}^{\mathrm{H}}=\left(E_{\mathrm{CASSCF}}-E_{\mathrm{HF}}\right) \\
& +\left(E_{\mathrm{FCI}}-E_{\mathrm{CASSCF}}\right)
\end{aligned}
$$

This expression for dynamic correlation energy actually coincides with the electron correlation energy definition suggested by Davidson some years earlier. ${ }^{49}$

Cioslowski's, Handy's and Ludeña's are schemes that afford the separation of dynamic and nondynamic correlation energies. There are very few other quantitative indicators of electron correlation and, to the best of our knowledge, there is no other measure that can quantify the relative importance of both effects. Ugalde and coworkers suggested the use of the intracule densities to obtain a separation of dynamic and nondynamic correlation effects ${ }^{50}$ but their approach, in the spirit of Handy's, also relies on a user-defined wavefunction that contains nondynamic electron correlation effects.

It would be more convenient if the separation of dynamic and nondynamic correlation would not depend on the definition of a wavefunction containing only nondynamic correlation effects. First of all, such definition is always arbitrary and, in some cases (see the discussion on $\mathrm{H}_{2}, \mathrm{LiH}$ and $\mathrm{Li}_{2}$ below), difficult to construct. Besides, an approach that allows the separation of electron correlation is more convenient than an energy-decomposition scheme because it could be used to split the correlation contributions of other observables. Ideally, the measure should depend on very simple quantities. For instance, expressions based on natural orbital occupancies (NOO) would accomplish this goal and would be directly applicable to natural orbital and density matrix functional theories $^{51,52}$ as well as wavefunction $a b$ initio calculations. ${ }^{1}$ In addition, through appropriate transformations, the NOO-based measures of electron correlation could also be applied in density functional theory. ${ }^{53,54}$ One such measure is the deviation from idempotency (DFI) ${ }^{5,55}$ that uses NOO and accounts for nondynamic correlation effects. There is actually very few indicators of dynamic electron correlation available in the literature and, to our knowledge, there is no NOO-based dynamic correlation measure excepting for Ziesche's proposal, ${ }^{3}$ which uses NOO of orbitals close to the Fermi level but it is not continuous with respect to small changes of NOO.

\section{Dynamic and nondynamic separation}

In order to construct a physically-motivated separation of dynamic and nondynamic correlation effects we will use the second- and first-order reduced density matrices (2-RDM and 1-RDM) in a natural orbital representation, respectively:

$$
\begin{gathered}
\rho_{1}\left(\mathbf{1} ; \mathbf{1}^{\prime}\right)=\sum_{i} n_{i} \phi_{i}^{*}\left(\mathbf{1}^{\prime}\right) \phi_{i}(\mathbf{1}) \\
\rho_{2}\left(\mathbf{1}, \mathbf{2} ; \mathbf{1}^{\prime}, \mathbf{2}^{\prime}\right)=\sum_{i j k l}{ }^{2} D_{k l}^{i j} \phi_{i}^{*}\left(\mathbf{1}^{\prime}\right) \phi_{j}^{*}\left(\mathbf{2}^{\prime}\right) \phi_{k}(\mathbf{1}) \phi_{l}(\mathbf{2})
\end{gathered}
$$

where $\phi_{i}(\mathbf{1})$ is a natural orbital and $n_{i}$ its occupancy, $\mathbf{1} \equiv\left(\vec{r}_{1}, \sigma_{1}\right)$, and ${ }^{2} D_{k l}^{i j}$ is the matrix representation of the 2-RDM (2-DM hereafter) and it should contain the electron correlation information of the system. ${ }^{56}$ The Hartree-Fock-like approximation of the 2-DM becomes a very simple function in terms of NOO, ${ }^{5}$

$$
\left({ }^{2} D^{\mathrm{HFL}}\right)_{k l}^{i j}=n_{i} n_{j}\left(\delta_{i k} \delta_{j l}-\delta_{i l} \delta_{j k}\right)
$$

the expression being exact for single-determinant wavefunctions. Let us define a pseudo Hartree-Fock (HF) 2-DM in the natural orbital representation

$$
\left({ }^{2} D^{\mathrm{PHF}}\right)_{k l}^{i j}=\xi_{i j k l}\left(\delta_{i k} \delta_{j l}-\delta_{i l} \delta_{j k}\right),
$$

where $\xi_{i j k l}$ equals 1 if $i, j, k$ and $l$ orbitals are below the Fermi level, zero otherwise. This matrix construction is very close to the approach followed by Scuseria and co-workers in the context of projected Hartree-Fock method. ${ }^{57,58}$ The difference with respect to the actual HF 2-DM is that the pseudo-HF matrix is defined in terms of the natural orbitals of the correlated calculation whereas the actual HF 2-DM is defined in terms of the canonical HF orbitals (i.e., the expression looks like eqn (6) but $i, j$, etc. refer to canonical HF orbitals). We have checked that energy-wise the difference between both matrices is fairly small and, for practical reasons, one can take the latter as a matrix free of electron correlation effects. In this sense, the difference between the actual 2-DM and the pseudo-HF one is a matrix that contains electron correlation effects,

$$
C_{k l}^{i j}={ }^{2} D_{k l}^{i j}-\left({ }^{2} D^{\mathrm{PHF}}\right)_{k l}^{i j} .
$$

In order to extract the electron correlation information from these matrices let us decompose $\mathbf{C}$ as

$$
\mathbf{C}=\Lambda+\Gamma,
$$

where,

$$
\begin{aligned}
& \Lambda_{k l}^{i j}=\left(n_{i} n_{j}-\xi_{i j k l}\right)\left(\delta_{i k} \delta_{j l}-\delta_{i l} \delta_{j k}\right) \\
& \Gamma_{k l}^{i j}={ }^{2} D_{k l}^{i j}-\left(n_{i} n_{j}\right)\left(\delta_{i k} \delta_{j l}-\delta_{i l} \delta_{j k}\right)
\end{aligned}
$$




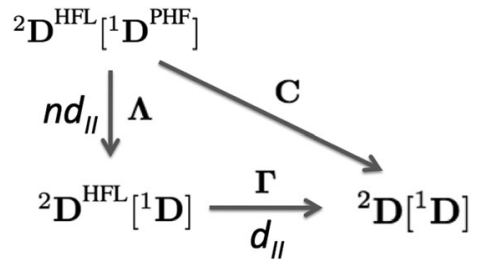

Fig. 2 Electron correlation diagram for 2-DM in terms of 1-DM.

$\Lambda$ is an antisymmetric diagonal matrix that measures the pairwise deviation of NOO from a single-determinant picture and $\Gamma$ is the cumulant matrix. ${ }^{59,60}$ The decomposition of $\mathbf{C}$ is sketched in the diagram of Fig. 2, corresponding to the two steps from the pseudo-HF 2-DM to the actual 2-DM. In this diagram ${ }^{1} \mathbf{D}^{\mathrm{PHF}}$ is the pseudo-HF 1-DM that we use to obtain ${ }^{2} \mathbf{D}^{\mathrm{PHF}}$ in eqn (6). The arrow pointing downwards represents the change in the 2-DM approximation due to the use of exact NOOs instead of the pseudo-HF ones. This relaxation process is expected to retrieve nondynamic correlation effects and is accounted by A, i.e., ${ }^{2} \mathbf{D}^{\mathrm{PHF}}={ }^{2} \mathbf{D}^{\mathrm{HFL}}\left[{ }^{1} \mathbf{D}^{\mathrm{PHF}}\right]$. The arrow that points from ${ }^{2} \mathbf{D}^{\mathrm{HFL}}\left[{ }^{1} \mathbf{D}\right]$, eqn (5), to the exact 2-DM represents the dynamic correlation effects contained in $\Gamma$. Notice that the exact electron density is retrieved by one-coordinate reduction of both ${ }^{2} \mathbf{D}^{\mathrm{HFL}}\left[{ }^{1} \mathbf{D}\right]$ and ${ }^{2} \mathbf{D}\left[{ }^{1} \mathbf{D}\right]$ but it is not obtained from ${ }^{2} \mathbf{D}^{\mathrm{HFL}}\left[{ }^{1} \mathbf{D}^{\mathrm{PHF}}\right]$. Since nondynamic correlation is expected to produce global changes to the electron density and dynamic correlation rather small local ones, it is only natural to assign $\Lambda$ and $\Gamma$ as the matrices accounting for nondynamic and dynamic correlation effects, respectively. Notwithstanding, notice that the trace of $\Gamma$-that corresponds to the deviation from idempotency-and the Frobenius norm of this matrix ${ }^{8}$ actually correspond to two well-known measures of non-dynamic correlation and, therefore, it is not obvious how to extract the pertinent correlation information from these matrices.

Indeed, eqn (8) provides a decomposition of a matrix that contains electron correlation into dynamic and nondynamic parts, however, how do we extract the information about electron correlation from these matrices? In order to provide an answer, we will use a two-electron model system.

\section{Two-electron model}

Let us analyze the correlation matrices defined in the latter section on a simple model system (MS) consisting of a singlet two-electron system. We will use a minimal basis (two orbitals) because the sign of the natural orbitals amplitude is completely determined in this case ${ }^{61}$ and, therefore, the 2-RDM that can be explicitly written in terms of natural occupancies. ${ }^{62}$ Let us consider a separation of the physical space into two symmetric regions $F$ and $F^{\prime}$, each containing an average of one electron. The electron fluctuation between these regions can be measured through the covariance: ${ }^{63,64}$

$$
V_{F, F^{\prime}}=\left\langle\left(\hat{N}_{F}-\bar{N}_{F}\right)\left(\hat{N}_{F^{\prime}}-\bar{N}_{F^{\prime}}\right)\right\rangle=\left\langle\hat{N}_{F} \hat{N}_{F^{\prime}}\right\rangle-1
$$

where $\hat{N}_{F}$ is the particle number operator acting on region $F, \bar{N}_{F}$ is average number of electrons in $F$ and $\left\langle\hat{N}_{F} \hat{N}_{F^{\prime}}\right\rangle$ is computed using the 2-DM. Electron correlation in this two-electron model can be measured by comparing the electron fluctuation between $F$ and $F^{\prime}$ using the 2-DM matrices given in Fig. 2, i.e.,

$$
\begin{gathered}
I_{\mathrm{ND}}^{\mathrm{MS}}=V_{F, F^{\prime}}\left[{ }^{2} \mathbf{D}^{\mathrm{HFL}}\left[{ }^{1} \mathbf{D}\right]\right]-V_{F, F^{\prime}}\left[{ }^{2} \mathbf{D}^{\mathrm{HFL}}\left[{ }^{1} \mathbf{D}^{\mathrm{PHF}}\right]\right] \\
=\sum_{i j k l} \sum_{\sigma \sigma^{\prime}} \Lambda_{k^{\sigma} l^{\sigma^{\prime}}}^{i^{\sigma} \sigma^{\prime}} S_{i k}^{F} S_{j l}^{F^{\prime}} \\
I_{\mathrm{D}}^{\mathrm{MS}}=V_{F, F^{\prime}}\left[{ }^{2} \mathbf{D}\right]-V_{F, F^{\prime}}\left[{ }^{2} \mathbf{D}^{\mathrm{HFL}}\left[{ }^{1} \mathbf{D}\right]\right] \\
=\sum_{i j k l} \sum_{\sigma \sigma^{\prime}} \Gamma_{k^{\sigma} l l^{\prime}}^{\sigma^{\sigma} \sigma^{\sigma^{\prime}}} S_{i k}^{F} S_{j l}^{F^{\prime}}
\end{gathered}
$$

where $S_{i k}^{F}$ is the overlap between two natural orbitals in the three-dimensional region of fragment $F$, i.e.,

$$
S_{i k}^{F}=\int_{F} \phi_{i}^{*}(\vec{r}) \phi_{k}(\vec{r}) \mathrm{d} \vec{r}
$$

Due to symmetry restrictions $S_{i i}^{F}=S_{i i}^{F^{\prime}}=1 / 2$ and it has been proved $^{65}$ that $S_{12}^{F} S_{21}^{F^{\prime}} \approx-\frac{1}{4}$. Therefore, the formulae can be simplified to

$$
\begin{gathered}
I_{\mathrm{D}}^{\mathrm{MS}}=n^{1 / 2}(1-n)^{1 / 2}-2 n(1-n) \\
I_{\mathrm{ND}}^{\mathrm{MS}}=2 n(1-n)
\end{gathered}
$$

where $n$ is the natural orbital occupancy of either the bonding or antibonding orbital. These indices are plotted in Fig. 3 against the occupation value. Simple inspection reveals that all the indices are zero for full or vanishing occupations, but the dynamic indicator attains larger values than the nondynamic one in the vicinity of these extreme occupancies. The nondynamic indicator is maximal when each electron is equally distributed between the two orbitals, whereas the dynamic counterpart is minimal in this situation. The dynamic indicator peaks at $n \approx 0.067$ and $n \approx 0.933$. All these features are in line with trends that one would expect from dynamic and nondynamic indicators.

\section{Natural orbital formulation}

In order to construct a general natural-orbital based decomposition of electron correlation in the following we assume that

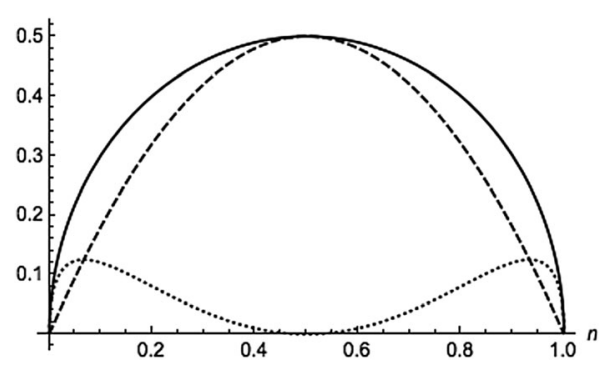

Fig. 3 Total (solid), dynamic (dotted) and nondynamic (dashed) electron correlation indicators for a homonuclear minimal-basis singlet twoelectron model against the orbital occupation. 
the correlation of a molecule can be retrieved by summing up the individual contributions of each orbital as calculated using eqn (17) and (18), i.e.,

$$
\begin{gathered}
I_{\mathrm{D}}=\frac{1}{4} \sum_{\sigma, i}\left[n_{i}^{\sigma}\left(1-n_{i}^{\sigma}\right)\right]^{1 / 2}-\frac{1}{2} \sum_{\sigma, i} n_{i}^{\sigma}\left(1-n_{i}^{\sigma}\right) \\
I_{\mathrm{ND}}=\frac{1}{2} \sum_{\sigma, i} n_{i}^{\sigma}\left(1-n_{i}^{\sigma}\right)
\end{gathered}
$$

where $n_{i}^{\sigma}$ is the occupation of the spin-natural orbital $i$ with spin $\sigma . I_{\mathrm{ND}}$ coincides with the DFI of the first-order density matrix, which is a well-known measure of nondynamic correlation and it is also linked to the number of effectively unpaired electrons in singlet molecules. ${ }^{66-70}$ The summation of both quantities gives rise to a total correlation index:

$$
I_{\mathrm{T}}=I_{\mathrm{D}}+I_{\mathrm{ND}}=\frac{1}{4} \sum_{\sigma, i}\left[n_{i}^{\sigma}\left(1-n_{i}^{\sigma}\right)\right]^{1 / 2}
$$

Unlike the previous formulation, eqn (8)-(10), which depend on the 2-DM, the expressions in eqn (19)-(21) provide simple measures of dynamic, nondynamic and total electron correlation in terms of natural orbital occupancies. Therefore, these expressions are more versatile and can be applied to all sort of $a b$ initio methods, density matrix functional theory (DMFT) $)^{51,52,71}$ and, DFT with fractional occupancies (or regular DFT by mapping orbital occupancies into Kohn-Sham orbital energies ${ }^{53}$ ). In addition, these formulae can be naturally decomposed into orbital contributions and, upon multiplication of orbital amplitudes, they afford local descriptors of total, dynamic and nondynamic correlation. These descriptors can be used to measure the local importance of dynamic correlation effects and hence be employed in the development of local methods. ${ }^{43}$

$I_{\mathrm{ND}}$ is defined in the interval $[0, N / 2]$, while $I_{\mathrm{D}}$ takes values in $[0, \infty]$ because the sum $\sum_{i} n_{i}{ }^{1 / 2}$ is not bounded above. Indeed, for an extreme case of infinite occupancies going to zero, the latter sum might diverge. Although this result is highly unpleasant, it merely has any effect for real systems where orbital occupancies never reach such situation.

In the Löwdin-Shull wavefunction the coefficients of the CI expansion take a very simple form in terms of natural occupancies, $2 c_{i}^{2}=n_{i}$. Therefore, one can easily study these indicators under different electron correlation regimes. ${ }^{60}$ A typical dynamic correlation case is characterized by a single dominant configuration, $c_{0} \approx 1$ (thus $c_{i}^{2} \approx 0 \forall i \neq 0$ ). For this system, eqn (20) goes quickly to zero, and the first term of eqn (19) dominates over the second one. Therefore, $I_{\mathrm{T}} \approx I_{\mathrm{D}}$, as one would expect. As a prototype of strong nondynamic correlation effects we analyze the degeneracy between two electron configurations, which for a two-electron system corresponds to $c_{0}=-c_{1}=\frac{1}{\sqrt{2}}$ (and $c_{i}^{2}=0 \forall i>1$ ). In this situation $I_{\mathrm{ND}}$ attains the maximum value and $I_{\mathrm{D}}=0$, in agreement with our prediction.

\section{Numerical examples}

We have performed numerical evaluations of the indices for a series of illustrative systems to validate the formulae derived from the two-electron model. FCI calculations with a cc-pVTZ basis set have been performed with a modified version of the program of Knowles and Handy ${ }^{72}$ for the dissociation of $\mathrm{H}_{2}$ and $\mathrm{LiH}$, for the planar potential energy surface of $\mathrm{H}_{4}$ and the isoelectronic series of He and Be. Full-valence CASSCF calculations have been performed with Gaussian 09 package $^{73}$ in order to obtain Handy's correlation energy decomposition (eqn (2)) for $\mathrm{H}_{2}$ and $\mathrm{H}_{4}$. The same program has been used to obtain CISD/cc-pVTZ natural orbitals for $\mathrm{Be}_{2}, \mathrm{CO}, \mathrm{F}_{2}, \mathrm{HF}, \mathrm{Li}_{2}$, $\mathrm{LiH}$ and $\mathrm{N}_{2}$, in order to calculate $I_{\mathrm{ND}}$ and $I_{\mathrm{D}}$, which are compared against the $E_{\mathrm{ND}}$ and $E_{\mathrm{D}}$ values published by Handy. ${ }^{48}$

The values of $I_{\mathrm{D}}, I_{\mathrm{ND}}, E_{\mathrm{D}}$ and $E_{\mathrm{ND}}$ along the dissociation of $\mathrm{H}_{2}$ are depicted in Fig. 4. At the equilibrium distance, the nondynamic correlation is barely zero and the most important contribution comes from the dynamic correlation. $I_{\mathrm{ND}}$ presents a sigmoidal growth as the bond stretches and it reaches the maximum value of 0.5 at dissociation. $I_{\mathrm{D}}$ slightly increases upon dissociation peaking at around 1.2 ̊. From this point on, the index decays to zero because, as the interaction between electrons decreases, the presence of isolated electrons cannot give rise to dynamic correlation. $E_{\mathrm{ND}}$ and $E_{\mathrm{D}}$ show qualitatively the same trend than $I_{\mathrm{ND}}$ and $E_{\mathrm{ND}}$, i.e., $E_{\mathrm{ND}}$ dominates over $E_{\mathrm{D}}$ and attains a maximum value at large distances whereas $E_{\mathrm{D}}$ has larger values than $E_{\mathrm{ND}}$ close to the equilibrium distance and decays to zero as the $\mathrm{H}_{2}$ molecule stretches. However, $E_{\mathrm{D}}$ has a maximum at shorter interatomic distance and shows smaller numbers than $I_{\mathrm{D}}$ (with respect to the total values). We attribute these differences to the fact that $E_{\mathrm{D}}$ is calculated assuming that a CAS $(2,2)$ calculation of a two-electron system will include no dynamical correlation. This example puts forward the difficulty of using reference wavefunctions that do not include dynamic correlation.

The isoelectronic series of Be and He have been widely used to calibrate dynamic and nondynamic correlation effects. ${ }^{45,46}$ As the effective nuclear charge increases the electron correlation
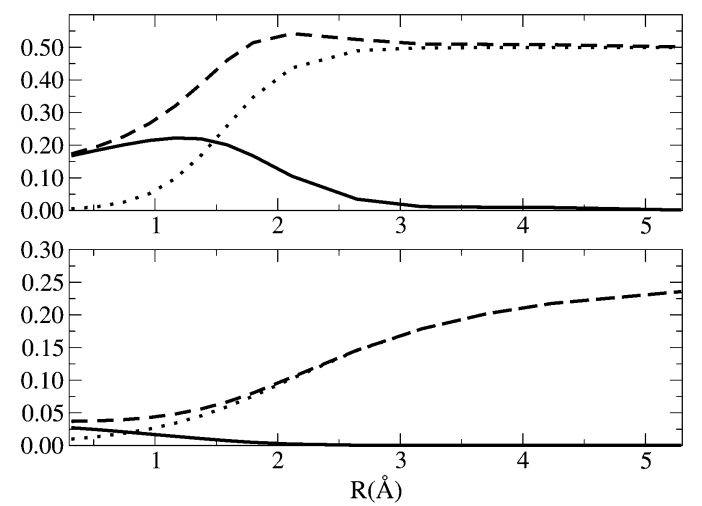

Fig. $4 I_{\mathrm{D}}$ (solid line) $I_{\mathrm{ND}}$ (dotted line) and $I_{\mathrm{T}}$ (dashed line) [top], $E_{\mathrm{D}}$ (solid line), $E_{\mathrm{ND}}$ and $E_{\mathrm{CORR}}$ (dashed line) [bottom] along the $\mathrm{H}_{2}$ dissociation curve. The equilibrium distance is $0.7 \AA$. Energy units are Hartrees. 

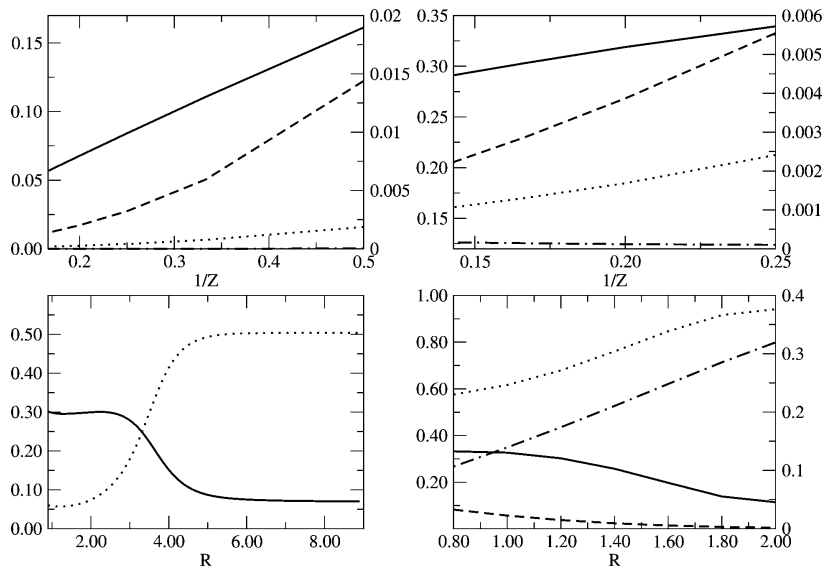

Fig. 5 From left to right and top to bottom: ID (solid line), / ND (dotted line), $\left|E_{\mathrm{D}}^{(1)}\right| / E_{\mathrm{FCl}}$ (dashed line) and $\left|E_{\mathrm{ND}}^{(1)}\right| / E_{\mathrm{FCl}}$ (dot-dashed line) along (i) the $\mathrm{He}$ isoelectronic series as a function of $1 / Z$, (ii) the Be isoelectronic series as a function of $1 / Z$, (iii) the $\mathrm{LiH}$ dissociation as a function of $R_{\mathrm{LiH}}(\AA)$ and (iv) $I_{\mathrm{D}}$ (solid line), $I_{\mathrm{ND}}$ (dotted line), $\left|E_{\mathrm{D}}^{\mathrm{H}}\right|$ (dashed line) and $\left|E_{\mathrm{ND}}^{\mathrm{H}}\right|$ (dot-dashed line) for the dissociation of the $D_{4 h}$ geometry of $\mathrm{H}_{4}$ as we increase the distance between the $\mathrm{H}$ atom and the center of mass, $R(\AA)$. Left $y$-axis is used for $I_{D}$ and $I_{\mathrm{ND}}$, whereas right $y$-axis is used for $E_{\mathrm{D}}$ and $E_{\mathrm{ND}}$.

decreases because the electron distribution concentrates around the nuclei and vice versa. For the He isoelectronic series the dynamic correlation is far more important than the nondynamic one due to the absence of orbital degeneracies, as illustrated by Fig. 5. The $2 \mathrm{~s}$ and $2 \mathrm{p}$ orbitals of Be-like ions are near degenerate and the HOMO-LUMO gap is thus very small. As $Z$ increases the gap increases but the relative gap (the gap divided by the average energy of the orbitals) actually decreases. Gill has called this correlation type B (nondynamic) correlation, whereas the correlation due to the absolute degeneracy of the gap is referred as type A (nondynamic) correlation. ${ }^{74}$ The results in Fig. 5 show that the DFI can identify the nondynamic correlation linked to type A, but it does not recognize the type B nondynamic correlation. Despite the presence of degeneracies, $I_{\mathrm{D}}$ is far more important than $I_{\mathrm{ND}}$. In order to compare these results with a correlation energy decomposition we should scale $E_{\mathrm{D}}$ and $E_{\mathrm{ND}}$ with respect to $E_{\mathrm{FCI}}$. The energy and, therefore, the correlation energy are highly sensible to the external potential. Hence, a crude inspection of $E_{\text {CORR }}$ does not inform about the relative correlation effects in a system with varying external potential unless an appropriate scaling is performed. Fig. 5 contains Cioslowski's decomposition results presented by Ludeña and coworkers, ${ }^{47}$ which show similar trends to $I_{\mathrm{D}}$ and $I_{\mathrm{ND}}$; the most notable exception being the small nondynamic correlation energy fraction attributed to the Be series. In Fig. 5 we also include the dissociation of $\mathrm{LiH}$, where both $I_{\mathrm{D}}$ and $I_{\mathrm{ND}}$ exhibit a similar shape as in $\mathrm{H}_{2}$.

In Fig. 5 we examine the singlet $\mathrm{H}_{4}$ planar potential energy surface ${ }^{75}$ by collecting the $D_{4 \mathrm{~h}}$ structures as a function of $R$, the distance between $\mathrm{H}$ and the center of mass (see Fig. 6). These geometries present degenerate $b_{3 u}$ and $b_{2 u}$ orbitals that prompt large nondynamic correlation effects as reflected by the values of $I_{\mathrm{ND}}\left(\left|E_{\mathrm{ND}}^{\mathrm{H}}\right|\right)$, which are bigger than the $I_{\mathrm{D}}\left(\left|E_{\mathrm{D}}^{\mathrm{H}}\right|\right)$ ones for all the structures studied. The more stretched the $\mathrm{H}_{4}$ structure,

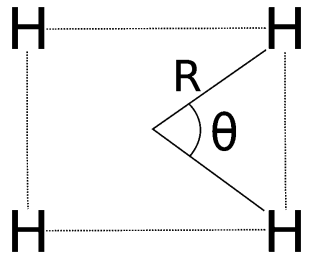

Fig. 6 The $D_{4 h} / D_{2 h}$ potential energy surface of $\mathrm{H}_{4}$ is given in terms of two coordinates: $R$ and $\theta$.

the larger the nondynamic correlation effects, whereas dynamic correlation is most notorious for constricted geometries. The transition from $D_{4 \mathrm{~h}}$ to $D_{2 \mathrm{~h}}$ structures (that is controlled by the angle $\theta$ between two contiguous $\mathrm{H}$ atoms and the center of mass) can be used to see the change from nondynamic-controlled region (near the $D_{4 \mathrm{~h}}$ geometries) to a region of predominant dynamic correlation. As $R$ increases, the values of $I_{\mathrm{D}}$ and $\left|E_{\mathrm{D}}^{\mathrm{H}}\right|$ decrease and the plots as a function of $\theta$ show a minimum at the $D_{4 \mathrm{~h}}$ structure and become flatter, in agreement with the small dynamic correlation expected at stretched geometries (see Fig. 7 and 8). $I_{\mathrm{ND}}$ and $\left|E_{\mathrm{ND}}^{\mathrm{H}}\right|$ show exactly the opposite profiles, with largest values at the $D_{4 \mathrm{~h}}$ geometries and flatter profiles for large $R$.

Finally, Table 1 collects a few diatomic molecules at the equilibrium distance $\left(R_{\mathrm{e}}\right)$ and at a stretched geometry $\left(1.5 R_{\mathrm{e}}\right)$. As expected, the nondynamic correlation indices increase upon bond length elongation with only a few exceptions. $\mathrm{Be}_{2}$ is an exception due to the unusually large nondynamic correlation nature at the ground state distance, as confirmed by the large $I_{\mathrm{ND}}$ value at equilibrium that barely changes for the stretched bond length. $\mathrm{LiH}$ and $\mathrm{Li}_{2}$ show a $I_{\mathrm{ND}}$ reduction upon stretching the internuclear distance, whereas $\left|E_{\mathrm{ND}}^{\mathrm{H}}\right|$ remains constant. This feature was recognized by Handy and coworkers, who suggested an alternative electron correlation energy partition, consisting of a CASSCF calculation with an active space such that the angular correlation is not incorporated. ${ }^{48}$ The results
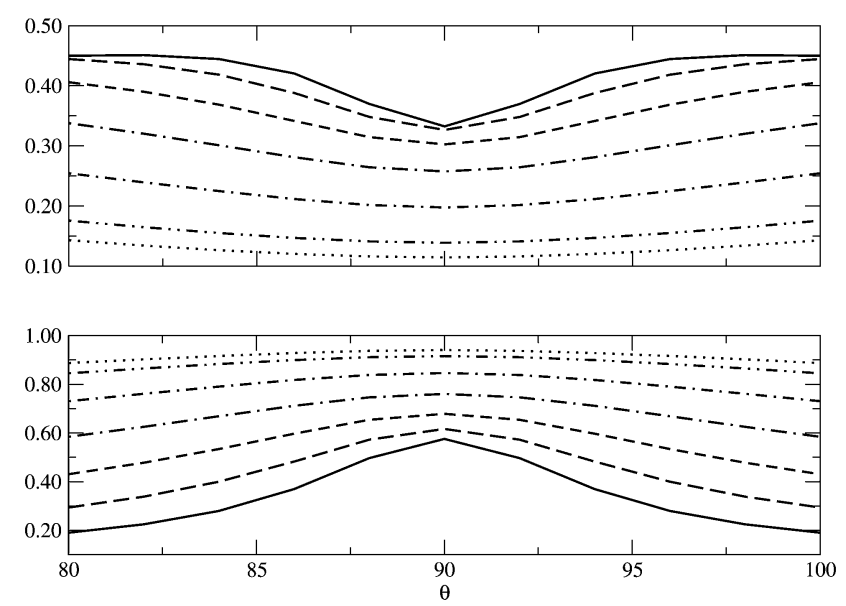

Fig. $7 I_{\mathrm{D}}$ (top) and $I_{\mathrm{ND}}$ (bottom) as a function of $\theta$, the angle between two contiguous $\mathrm{H}$ atoms and the center of mass in the $\mathrm{H}_{4}$ molecule, for different $R$ (see Fig. 6). The solid lines represent $R=0.8 \AA$ and the dotted lines $R=2.0 \AA$, the other lines in between correspond to the structures of $R=1.0,1.2,1.4,1.6$ and $1.8 \AA$. 

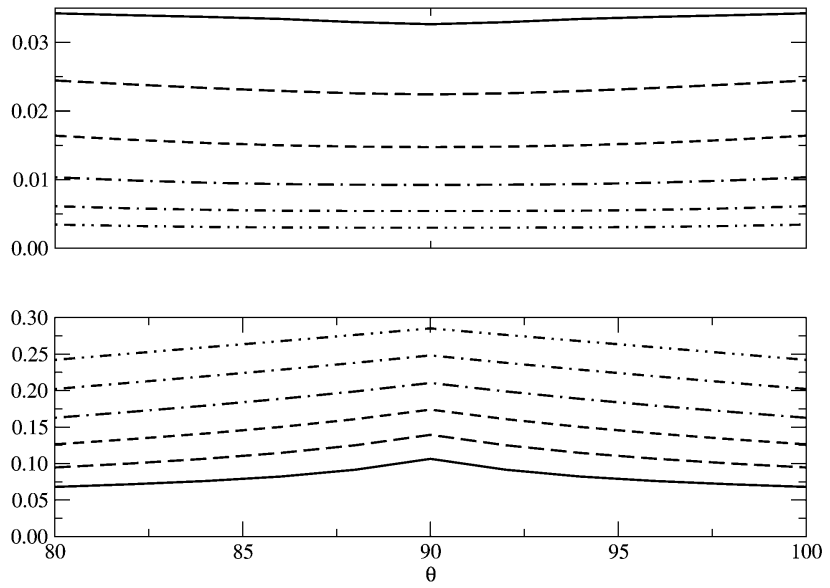

Fig. $8\left|E_{\mathrm{D}}^{\mathrm{H}}\right|$ (top) and $\left|E_{N \mathrm{HD}}^{\mathrm{H}}\right|$ (bottom) as a function of $\theta$, the angle between two contiguous $\mathrm{H}$ atoms and the center of mass in the $\mathrm{H}_{4}$ molecule, for different $R$ (see Fig. 6). The solid lines represent $R=0.8 \AA$ and the dotted lines $R=2.0 \AA$, the other lines in between correspond to the structures of $R=1.0,1.2,1.4,1.6$ and $1.8 \AA$. Units are Hartrees.

Table $1 \mathrm{CISD} / \mathrm{cc}-\mathrm{pVTZ} I_{\mathrm{D}}, I_{\mathrm{ND}}, E_{\mathrm{D}}$ and $E_{\mathrm{ND}}$ values for a series of diatomic molecules at its ground state geometry $\left(R_{\mathrm{e}}\right)$ and at the $1.5 R_{\mathrm{e}}$ interatomic separation. $I_{\mathrm{D}}, I_{\mathrm{ND}}$ units are electrons and $E_{\mathrm{D}}$ and $E_{\mathrm{ND}}$ units are Hartrees

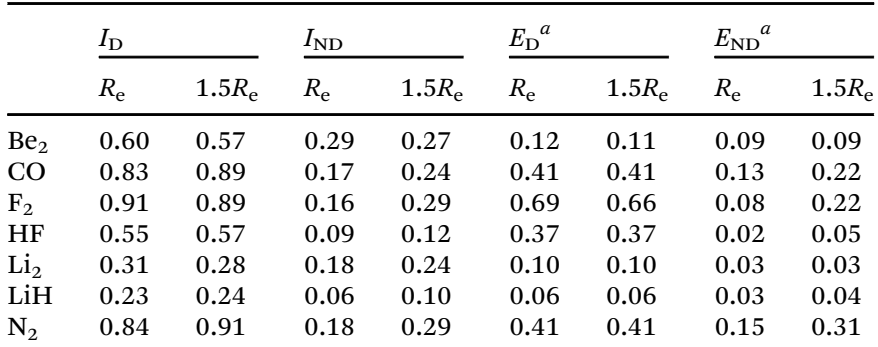

${ }^{a} E_{\mathrm{D}}$ and $E_{\mathrm{ND}}$ are taken ref. 48.

obtained with this alternative partition do show some increase of $\left|E_{\mathrm{ND}}^{\mathrm{H}}\right|$ for $\mathrm{LiH}$ and $\mathrm{Li}_{2}$ due to bond length elongation, ${ }^{48}$ however, they put forward the limitation of the CASSCF wavefunction as a reference containing no dynamic correlation.

\section{Conclusions}

In this paper we have developed physically-sound electron correlation matrices that account for total (eqn (8)), nondynamic (eqn (9)) and dynamic (eqn (10)) correlation effects and depend on the pair density. These expressions are applied to a minimal-basis two-fragment two-electron model at different inter-fragment separations to afford dynamic (eqn (19)), nondynamic (eqn (20)) and total (eqn (21)) electron correlation indicators in terms of natural orbital occupancies. Unlike other existing indicators of electron correlation, they neither depend upon the definition of wavefunctions containing only dynamic or nondynamic correlation effects, nor they need the calculation of the exact wavefunctions. The indicators here developed do not rely on reference wavefunctions and can be applied to any method to analyze the effect of dynamic and nondynamic correlation provided that natural orbital occupancies are available. The electron correlation indicators have been analyzed in a set of representative examples providing different dynamic and nondynamic electron correlation regimes. Results are also compared against existing electron correlation measures, demonstrating the validity of the new expressions.

The latter expressions can be applied to all sort of $a b$ initio methods, DMFT and, ensemble DFT with fractional occupancies $^{76}$ (or DFT by mapping orbital occupancies into Kohn-Sham orbital energies ${ }^{53}$ ). In addition, these formulae can be naturally decomposed into orbital contributions and, upon multiplication of orbital amplitudes, they afford local descriptors of total, dynamic and nondynamic correlation. These descriptors measure the local importance of dynamic correlation and hence can be used in the development of local methods. ${ }^{43}$ Such possibility is currently explored in our lab and will be the subject of a future work.

\section{Acknowledgements}

This research has been funded by the Spanish MINECO Projects No. CTQ2014-52525-P and CTQ2014-59212-P, and the Basque Country Consolidated Group Project No. IT588-13. ERC acknowledges funding from the European Union's Horizon 2020 research and innovation programme under the Marie Sklodowska-Curie grant agreement No. 660943. E. M. acknowledges fruitful discussions with Profs Savin, Szabados and Cioslowski.

\section{References}

1 C. Hättig, W. Klopper, A. Köhn and D. P. Tew, Chem. Rev., 2012, 112, 4-74.

2 D. Cremer, Mol. Phys., 2001, 99, 1899-1940.

3 P. Ziesche, THEOCHEM, 2000, 527, 35-50.

4 D. P. Tew, W. Klopper and T. Helgaker, J. Comput. Chem., 2007, 28, 1307-1320.

5 P.-O. Löwdin, Phys. Rev., 1955, 97, 1474-1489.

6 W. Kutzelnigg, G. Del Re and G. Berthier, Phys. Rev., 1968, 172, 49.

7 K. Raghavachari and J. B. Anderson, J. Phys. Chem., 1996, 100, 12960-12973.

8 T. Juhász and D. A. Mazziotti, J. Chem. Phys., 2006, 125, 174105.

9 D. Alcoba, R. Bochicchio, L. Lain and A. Torre, J. Chem. Phys., 2010, 133, 144104.

10 J. T. Skolnik and D. A. Mazziotti, Phys. Rev. A: At., Mol., Opt. Phys., 2013, 88, 032517.

11 O. Sinanoğlu, Adv. Chem. Phys., 1964, 6, 315-412.

12 R. J. Bartlett and J. F. Stanton, Rev. Comput. Chem., 2007, 5, 65-169.

13 J. E. Lennard-Jones, J. Chem. Phys., 1952, 20, 1024.

14 G. C. Lie and E. Clementi, J. Chem. Phys., 1974, 60, 1275-1287.

15 T. J. Lee, J. E. Rice, G. E. Scuseria and H. F. Schaefer, Theor. Chim. Acta, 1989, 75, 81. 
16 T. J. Lee and P. R. Taylor, Int. J. Quantum Chem., 1989, 23, 199-207.

17 C. L. Janssen and I. M. B. Nielsen, Chem. Phys. Lett., 1998, 290, 290.

18 T. J. Lee, Chem. Phys. Lett., 2003, 372, 362-367.

19 J. Cioslowski, Theor. Chim. Acta, 1992, 81, 319-327.

20 K. Andersson, P.-A. Malmqvist and B. O. Roos, J. Chem. Phys., 1992, 96, 1218-1226.

21 R. J. Buenker, S. D. Peyerimhoff and W. Butscher, Mol. Phys., 1978, 35, 771-791.

22 D. A. Mazziotti, Phys. Rev. A: At., Mol., Opt. Phys., 2007, 76, 052502.

23 J. W. Snyder Jr and D. A. Mazziotti, Phys. Chem. Chem. Phys., 2012, 14, 1660-1667.

24 A. Savin, Int. J. Quantum Chem., 1988, 34, 59-69.

25 A. Savin, Density functional methods in chemistry, Springer, 1991, pp. 213-230.

26 A. Savin, On degeneracy, near-degeneracy and density functional theory. In Recent Developments of Modern Density Functional Theory, Elsevier, Amsterdam, 1996.

27 J. Jaramillo, G. E. Scuseria and M. Ernzerhof, J. Chem. Phys., 2003, 118, 1068-1073.

28 A. D. Becke, J. Chem. Phys., 1993, 98, 5648-5652.

29 A. D. Becke, J. Chem. Phys., 1993, 98, 1372-1377.

30 J. P. Perdew, M. Ernzerhof and K. Burke, J. Chem. Phys., 1996, 105, 9982-9985.

31 F. G. Cruz, K.-C. Lam and K. Burke, J. Phys. Chem. A, 1998, 102, 4911-4917.

32 H. Iikura, T. Tsuneda, T. Yanai and K. Hirao, J. Chem. Phys., 2001, 115, 3540-3544.

33 R. Baer, E. Livshits and U. Salzner, Annu. Rev. Phys. Chem., 2009, 61, 85.

34 A. V. Arbuznikov and M. Kaupp, J. Chem. Phys., 2014, 141, 204101.

35 T. M. Henderson, B. G. Janesko and G. E. Scuseria, J. Phys. Chem. A, 2008, 112, 12530-12542.

36 E. R. Johnson, J. Chem. Phys., 2014, 141, 124120.

37 A. D. Becke, J. Chem. Phys., 1988, 88, 2547-2553.

38 P. De Silva and C. Corminboeuf, J. Chem. Phys., 2015, $142,074112$.

39 S. Grimme and A. Hansen, Angew. Chem., Int. Ed., 2015, 54, 12308-12313.

40 K. Boguslawski, P. Tecmer, O. Legeza and M. Reiher, J. Phys. Chem. Lett., 2012, 3, 3129-3135.

41 A. Raeber and D. A. Mazziotti, Phys. Rev. A: At., Mol., Opt. Phys., 2015, 92, 052502.

42 P. Pulay, Chem. Phys. Lett., 1983, 100, 151-154.

43 R. Zalesny, M. G. Papadopoulos, P. G. Mezey and J. Leszczynski, Linear-scaling techniques in computational chemistry and physics: Methods and applications, Springer Science + Business Media BV, 2011.

44 I. Shavitt, in Advanced Theories and Computational Approaches to the Electronic Structure of Molecules, ed. C. E. Dykstra, Springer, Netherlands, 1984, pp. 185-196.

45 J. Cioslowski, Phys. Rev. A: At., Mol., Opt. Phys., 1991, 43, 1223-1228.
46 E. Valderrama, E. V. Ludeña and J. Hinze, J. Chem. Phys., 1997, 106, 9227-9235.

47 E. Valderrama, E. V. Ludeña and J. Hinze, J. Chem. Phys., 1999, 110, 2343-2353.

48 D. K. W. Mok, R. Neumann and N. C. Handy, J. Phys. Chem., 1996, 100, 6225-6230.

49 E. Davidson, S. Hagstrom, S. Chakravorty, V. Umar and C. Fischer, Phys. Rev. A: At., Mol., Opt. Phys., 1991, 44, 7071.

50 E. Valderrama, J. Mercero and J. Ugalde, J. Phys. B: At. Mol. Phys., 2001, 34, 275.

51 M. Piris and J. Ugalde, Int. J. Quantum Chem., 2014, 114, 1169-1175.

52 K. Pernal and K. J. H. Giesbertz, Top. Curr. Chem., 2015, 368, 125.

53 M. Grüning, O. Gritsenko and E. Baerends, J. Chem. Phys., 2003, 118, 7183-7192.

54 C.-S. Wu and J.-D. Chai, J. Chem. Theory Comput., 2015, 11, 2003-2011.

55 V. H. Smith Jr, Theor. Chim. Acta, 1967, 7, 245.

56 C. A. Coulson, Rev. Mod. Phys., 1960, 32, 170.

57 G. E. Scuseria, C. A. Jiménez-Hoyos, T. M. Henderson, K. Samanta and J. K. Ellis, J. Chem. Phys., 2011, 135, 124108.

58 C. A. Jimenez-Hoyos, T. M. Henderson, T. Tsuchimochi and G. E. Scuseria, J. Chem. Phys., 2012, 136, 164109.

59 D. A. Mazziotti, Chem. Phys. Lett., 1998, 289, 419-427.

60 W. Kutzelnigg and D. Mukherjee, J. Chem. Phys., 1999, 110, 2800-2809.

61 K. Pernal and J. Cioslowski, J. Chem. Phys., 2004, 120, 5987-5992.

62 P.-O. Löwdin and H. Shull, Phys. Rev., 1956, 101, 1730-1739.

63 R. F. W. Bader and M. E. Stephens, Chem. Phys. Lett., 1974, 26, 445 .

64 E. Matito, M. Solà, P. Salvador and M. Duran, Faraday Discuss., 2007, 135, 325-345.

65 E. Matito, M. Duran and M. Solà, J. Chem. Educ., 2006, 83, 1243.

66 K. Takatsuka, T. Fueno and K. Yamaguchi, Theor. Chim. Acta, 1978, 48, 175-183.

67 V. N. Staroverov and E. R. Davidson, Chem. Phys. Lett., 2000, 330, 161-168.

68 M. Head-Gordon, Chem. Phys. Lett., 2003, 372, 508-511.

69 R. C. Bochicchio, A. Torre and L. Lain, Chem. Phys. Lett., 2003, 380, 486-487.

70 M. Head-Gordon, Chem. Phys. Lett., 2003, 380, 488-489.

71 D. A. Mazziotti, Chem. Rev., 2012, 112, 244-262.

72 P. Knowles and N. Handy, Comput. Phys. Commun., 1989, 54, 75.

73 M. J. Frisch, G. W. Trucks, H. B. Schlegel, G. E. Scuseria, M. A. Robb, J. R. Cheeseman, G. Scalmani, V. Barone, B. Mennucci, G. A. Petersson, H. Nakatsuji, M. Caricato, X. Li, H. P. Hratchian, A. F. Izmaylov, J. Bloino, G. Zheng, J. L. Sonnenberg, M. Hada, M. Ehara, K. Toyota, R. Fukuda, J. Hasegawa, M. Ishida, T. Nakajima, Y. Honda, O. Kitao, H. Nakai, T. Vreven, J. A. Montgomery, Jr., J. E. Peralta, F. Ogliaro, M. Bearpark, J. J. Heyd, E. Brothers, K. N. Kudin, V. N. Staroverov, R. Kobayashi, J. Normand, K. Raghavachari, 
A. Rendell, J. C. Burant, S. S. Iyengar, J. Tomasi, M. Cossi, N. Rega, J. M. Millam, M. Klene, J. E. Knox, J. B. Cross, V. Bakken, C. Adamo, J. Jaramillo, R. Gomperts, R. E. Stratmann, O. Yazyev, A. J. Austin, R. Cammi, C. Pomelli, J. W. Ochterski, R. L. Martin, K. Morokuma, V. G. Zakrzewski, G. A. Voth, P. Salvador, J. J. Dannenberg, S. Dapprich, A. D. Daniels, Ö. Farkas, J. B. Foresman,
J. V. Ortiz, J. Cioslowski and D. J. Fox, Gaussian 09 Revision D.01, Gaussian Inc., Wallingford CT, 2009.

74 J. W. Hollett and P. M. Gill, J. Chem. Phys., 2011, 134, 114111.

75 E. Ramos-Cordoba, X. Lopez, M. Piris and E. Matito, J. Chem. Phys., 2015, 143, 164112.

76 E. Fromager, Mol. Phys., 2015, 113, 419-434. 